\title{
Electroweak Precision Constraints on Vector-like Fermions
}

\author{
G. Cynolter and E. Lendvai \\ Theoretical Physics Research Group of Hungarian Academy of Sciences, \\ Eötvös University, Budapest, 1117 Pázmány Péter sétány 1/A, Hungary
}

\begin{abstract}
We calculate the oblique electroweak corrections and confront with the experiments in an extension of the Standard Model. The new fields added are a vector-like weak doublet and a singlet fermion. After electroweak symmetry breaking there is a mixing between the components of the new fields, but no mixing allowed with the standard fermions. Four electroweak parameters, $\hat{S}, \hat{T}, W$, $Y$ are presented in the formalism of Barbieri et al., these are the generalization of the Peskin-Takeuchi $S, T$, U's. The vector-like extension is slightly constrained, $\hat{T}$ requires the new neutral fermion masses not to be very far from each other, allowing higher mass difference for higher masses and smaller mixing. $\hat{S}, W, Y$ gives practically no constraints on the masses. This extension can give a positive contribution to $\hat{T}$, allowing a heavy Higgs boson in electroweak precision tests of the Standard Model.
\end{abstract}

\section{Introduction}

Vector-like fermions appear in several extensions of the Standard Model (SM). They are present in extra dimensional models with bulk fermions e.g [1], in little Higgs theories [2], in models of so called improved naturalness consistent with a heavy Higgs scalar [3], in simple fermionic models of dark matter [4, 5], in some dynamical models of supersymmetry breaking using gauge mediation, topcolor models [6], and were also considered as the solution to the discrepancy between $R_{b}$ and LEP2 measurements in the mid 90's [7]. Vector-like fermions were essential ingredients in a recent proposal, in which a nontrivial condensate of new vector-like fermions breaks the electroweak symmetry and provides masses for the standard particles [8]. The potential LHC signals of vector-like quarks were discussed in [9].

Any extension of the SM must face the tremendous success of the SM in high energy experiments, it must have evaded direct detection and fulfill the electroweak precision tests. If the scale of new physics is sufficiently high and the corrections are assumed to be universal then the new physics only affects the finite combinations of the gauge 
boson self-energies. These parameters (traditionally S,T,U [10]) are constrained by experiments [11. Barbieri et al. reconsidered the problem [12] and showed that there are indeed four relevant parameters $\hat{S}, \hat{T}, W, Y$, where $\hat{S}$ and $\hat{T}$ are related to the old parameters $S=4 s_{W}^{2} \hat{S} / \alpha, T=\hat{T} / \alpha$. $W$ and $Y$ are two new parameters, $U(\hat{U})$ is suppressed by the scale of new physics compared to $T(\hat{T})$. There are also other and more extended parameterizations are known [13].

In this letter we calculate the gauge boson vacuum polarization functions and precision electroweak observables for vector-like extensions of the SM, especially taking into account the mixing in the recently proposed fermion condensate model [8]. There are earlier results for extra vector-like quarks [14, 5] and detailed calculations for the $\rho$ parameter in the littlest Higgs model e.g. [15]. The new results in this paper are that we use different representations for the new fermions, give general formulae applicable to LEP2 measurements using the four parameters of [12] and constrain the fermion condensate model [8].

\section{Extension of the Standard Model with vector-like fermions}

We consider a simple extension of the SM based on non-chiral fermions. The new colorless fermions are an extra neutral weak $S U(2)$ singlet $\Psi_{S}(T=Y=0)$ and a doublet $\Psi_{D}=\left(\begin{array}{c}\Psi_{D}^{+} \\ \Psi_{D}^{0}\end{array}\right)$ with hypercharge 1. It is assumed that the new fermions are odd under a new $Z_{2}$ symmetry, while all the standard particles are even. This symmetry forbids mixings with standard fermions and the lightest new fermion is stable providing an ideal weakly interacting dark matter candidate. The purely fermionic part of the new Lagrangian is

$$
L_{\Psi}=i \bar{\Psi}_{D} D_{\mu} \gamma^{\mu} \Psi_{D}+i \bar{\Psi}_{S} \partial_{\mu} \gamma^{\mu} \Psi_{S}-m_{1} \bar{\Psi}_{D} \Psi_{D}-m_{2} \bar{\Psi}_{S} \Psi_{S}
$$

with Dirac masses $m_{1}, m_{2} . \Psi_{S}$ may have further interactions irrelevant for our analysis. $D_{\mu}$ is the covariant derivative

$$
D_{\mu}=\partial_{\mu}-i \frac{g}{2} \underline{\underline{W}} \underline{W}_{\mu}-i \frac{g^{\prime}}{2} B_{\mu}
$$

where $\underline{W}_{\mu}, B_{\mu}$ and $g, g^{\prime}$ are the usual weak gauge boson fields and couplings, respectively. In a renormalizable theory including the standard Higgs doublet $(H)$ additional Yukawa terms appear resulting a mixing between the new neutral fermions.

$$
L_{Y u k a w a}=\lambda_{m} \bar{\Psi}_{D} \Psi_{S} H+\lambda_{m}^{*} H^{\dagger} \bar{\Psi}_{S} \Psi_{D}
$$

In a version of the Standard Model [8], the Higgs boson is a composite state of the new fermions $\left(H=\bar{\Psi}_{S} \Psi_{D}\right)$ and these Yukawa terms (and additional contribution to $\Psi_{D}, \Psi_{S}$ mass ) generated by condensation from effective 4 -fermion interactions. 
When the Higgs (or the composite operator $\bar{\Psi}_{S} \Psi_{D}$ in [8]) develops a vacuum expectation value, $\langle H\rangle_{0}=\left(\begin{array}{l}0 \\ v\end{array}\right)$, with real $v$, non-diagonal mass terms are generated with $m_{3}=\left(\lambda_{m}+\lambda_{m}^{*}\right) v / 2$

$$
L_{\mathrm{mass}}=-m_{1} \bar{\Psi}_{D} \Psi_{D}-m_{2} \bar{\Psi}_{S} \Psi_{S}-m_{3}\left(\bar{\Psi}_{D}^{0} \Psi_{S}+\bar{\Psi}_{S} \Psi_{D}^{0}\right) .
$$

In [8] $m_{1}\left(m_{2}\right)$ get contributions from the condensates. The mass matrix of the new fermions must be diagonalized via unitary transformation to get physical mass eigenstates

$$
\begin{aligned}
& \Psi_{1}=c \Psi_{D}^{0}+s \Psi_{S}, \\
& \Psi_{2}=-s \Psi_{D}^{0}+c \Psi_{S},
\end{aligned}
$$

where $c=\cos \phi, s=\sin \phi, \phi$ is the mixing angle defined by

$$
2 m_{3}=\left(m_{1}-m_{2}\right) \tan 2 \phi
$$

The masses of the new neutral physical fermions $\Psi_{1}, \Psi_{2}$ are $M_{1,2}=\frac{1}{2}\left(m_{1}+m_{2} \pm \frac{m_{1}-m_{2}}{\cos 2 \phi}\right)$. The useful inverse relations are

$$
\begin{aligned}
& m_{1}=c^{2} M_{1}+s^{2} M_{2}, \\
& m_{2}=s^{2} M_{1}+c^{2} M_{2} .
\end{aligned}
$$

In the physical spectrum there is also a charged fermion $\Psi_{D}^{+}$, with mass $M_{+}=m_{1}$ (given by (7)). In the case of an elementary scalar field $\lambda_{m}$ is a free parameter. The mixing angle and the physical masses are basicly not constrained from the theory. In [8] gap equations determine the masses and the mixing angle. Applying further unitarity constraints one finds that one of the neutral masses is very close the charged mass and the mixing is rather weak [16].

The collider phenomenology and radiative corrections in the model are coming from the doublet kinetic term in (11) taking into account the mixing (5)

$$
\begin{aligned}
L^{I}= & \overline{\Psi_{D}^{+}} \gamma^{\mu} \Psi_{D}^{+}\left(\frac{g^{\prime}}{2} B_{\mu}+\frac{g}{2} W_{3 \mu}\right)+ \\
& +\left(c^{2} \bar{\Psi}_{1} \gamma^{\mu} \Psi_{1}+s^{2} \bar{\Psi}_{2} \gamma^{\mu} \Psi_{2}-s c\left(\bar{\Psi}_{1} \gamma^{\mu} \Psi_{2}+\bar{\Psi}_{2} \gamma^{\mu} \Psi_{1}\right)\right)\left(\frac{g^{\prime}}{2} B_{\mu}-\frac{g}{2} W_{3 \mu}\right)+ \\
& +\left[\frac{g}{\sqrt{2}} W_{\mu}^{+}\left(c \overline{\Psi_{D}^{+}} \gamma^{\mu} \Psi_{1}-s \overline{\Psi_{D}^{+}} \gamma^{\mu} \Psi_{2}\right)+\text { h.c. }\right] .
\end{aligned}
$$

We calculate the contribution to the electroweak precision observables from this renormalizable interaction. 


\section{$3 \quad$ Electroweak precision parameters}

Barbieri et al. showed [12] that if the scale of new physics is sufficiently higher than the LEP2 scale and the new physics affects only the vector boson self energies then the most general parameterization of new physics effects uses 4 parameters $\hat{S}, \hat{T}, W, Y$. These parameters are the generalizations of the Peskin-Takeuchi S,T,U parameters and defined from the transverse gauge boson vacuum polarization amplitudes

$$
\Pi_{a b}^{\mu \nu}\left(q^{2}\right)=g^{\mu \nu} \Pi_{a b}\left(q^{2}\right)+p^{\mu} p^{\nu} \text { terms }
$$

expanded up to the quadratic order $\left(a b=\left\{W^{+} W^{-}, W_{3} W_{3}, B B, W_{3} B\right\}\right)$

$$
\Pi_{a b}\left(q^{2}\right) \simeq \Pi_{a b}(0)+q^{2} \Pi_{a b}^{\prime}(0)+\frac{\left(q^{2}\right)^{2}}{2} \Pi_{a b}^{\prime \prime}(0)+\ldots
$$

. The relevant parameters are defined by

$$
\begin{aligned}
\left(g^{\prime} / g\right) \hat{S} & =\Pi_{W_{3} B}^{\prime}(0), \\
M_{W}^{2} \hat{T} & =\Pi_{W_{3} W_{3}}(0)-\Pi_{W^{+} W^{-}}(0), \\
2 M_{W}^{-2} Y & =\Pi_{B B}^{\prime \prime}(0), \\
2 M_{W}^{-2} W & =\Pi_{W_{3} W_{3}}^{\prime \prime}(0),
\end{aligned}
$$

here we use canonically normalized fields and $\Pi$ functions. The form factor $\hat{T}$ has custodial and $S U_{L}(2)$ breaking quantum numbers, while $\hat{S}$ respects custodial symmetry and breaks $S U_{L}(2) . \quad Y$ and $W$ are symmetric under both symmetries and they are important at the LEP2 energies. The result of the combined fit (excluding NuTeV) is shown in Table 1. from [12].

\begin{tabular}{|c|c|c|c|c|}
\hline & $10^{3} \hat{S}$ & $10^{3} \hat{T}$ & $10^{3} W$ & $10^{3} Y$ \\
\hline light Higgs & $0.0 \pm 1.3$ & $0.1 \pm 0.9$ & $0.1 \pm 1.2$ & $-0.4 \pm 0.8$ \\
\hline heavy Higgs & $-0.9 \pm 1.3$ & $2.0 \pm 1.0$ & $0.0 \pm 1.2$ & $-0.2 \pm 0.8$ \\
\hline
\end{tabular}

Table 1: Global fit of the electroweak precision parameters for a light $\left(M_{H}=115 \mathrm{GeV}\right)$ and a heavy $\left(M_{H}=800 \mathrm{GeV}\right)$ Higgs.

The calculation of the parameters is based on the general gauge boson vacuum polarization diagram with two non-degenerate fermions with masses $m_{a}$ and $m_{b}$. We use dimensional regularization and give the result for general $q^{2}$. The coupling constants are defined in the usual manner $L^{I} \sim V_{\mu} \bar{\Psi}\left(g_{V} \gamma^{\mu}+g_{A} \gamma_{5} \gamma^{\mu}\right) \Psi$.

$$
\begin{gathered}
\Pi\left(q^{2}\right)=\frac{1}{4 \pi^{2}}\left(\left(g_{V}^{2}+g_{A}^{2}\right) \tilde{\Pi}_{V+A}+\left(g_{V}^{2}-g_{A}^{2}\right) \tilde{\Pi}_{V-A}\right) \\
\tilde{\Pi}_{V+A}=-\frac{1}{2}\left(m_{a}^{2}+m_{b}^{2}-\frac{2}{3} q^{2}\right)\left(\operatorname{Div}+\ln \left(\frac{\mu^{2}}{m_{a} m_{b}}\right)\right)-\frac{\left(m_{a}^{2}-m_{b}^{2}\right)^{2}}{6 q^{2}}-\frac{1}{3}\left(m_{a}^{2}+m_{b}^{2}\right)+(15) \\
+\frac{5}{9} q^{2}-\frac{\left(m_{a}^{2}-m_{b}^{2}\right)^{3}}{12 q^{4}} \ln \left(\frac{m_{b}^{2}}{m_{a}^{2}}\right)+\frac{1}{3}\left(\frac{\left(m_{a}^{2}-m_{b}^{2}\right)^{2}}{q^{2}}+m_{a}^{2}+m_{b}^{2}-2 q^{2}\right) f\left(m_{a}^{2}, m_{b}^{2}, q^{2}\right)
\end{gathered}
$$


and

$$
\tilde{\Pi}_{V-A}=m_{a} m_{b}\left(\operatorname{Div}+\ln \left(\frac{\mu^{2}}{m_{a} m_{b}}\right)+2+\frac{\left(m_{a}^{2}-m_{b}^{2}\right)}{2 q^{4}} \ln \left(\frac{m_{b}^{2}}{m_{a}^{2}}\right)-2 f\left(m_{a}^{2}, m_{b}^{2}, q^{2}\right)\right) .
$$

The function $f\left(m_{a}^{2}, m_{b}^{2}, q^{2}\right)$ is given by

$$
f\left(m_{a}^{2}, m_{b}^{2}, q^{2}\right)=\left\{\begin{array}{cc}
\sqrt{\Delta} \operatorname{arctanh}^{-1}\left(\frac{\sqrt{\Delta} q^{2}}{q^{2}-\left(m_{a}+m_{b}\right)^{2}}\right) & q<\left|m_{a}-m_{b}\right| \\
-\sqrt{-\Delta} \arctan \left(\frac{\sqrt{-\Delta} q^{2}}{q^{2}-\left(m_{a}+m_{b}\right)^{2}}\right) & \left|m_{a}-m_{b}\right|<q \text { and } q<m_{a}+m_{b}, \\
\sqrt{\Delta} \operatorname{arccot}^{-1}\left(\frac{\sqrt{\Delta} q^{2}}{q^{2}-\left(m_{a}+m_{b}\right)^{2}}\right) & m_{a}+m_{b}<q
\end{array}\right.
$$

where we defined

$$
\Delta=1-2 \frac{m_{a}^{2}+m_{b}^{2}}{q^{2}}+\frac{\left(m_{a}^{2}-m_{b}^{2}\right)^{2}}{q^{4}}
$$

and Div $=1 / \epsilon+\ln 4 \pi-\gamma_{\epsilon}$ contains the usual divergent term in dimensional regularization.

The electroweak parameters depend on the values and derivatives of the $\Pi$ functions at $q^{2}=0$, the limits are given below.

$$
\begin{aligned}
\tilde{\Pi}_{V+A}(0)= & -\frac{1}{2}\left(m_{a}^{2}+m_{b}^{2}\right)\left(\operatorname{Div}+\ln \left(\frac{\mu^{2}}{m_{a} m_{b}}\right)\right)- \\
& -\frac{1}{4}\left(m_{a}^{2}+m_{b}^{2}\right)-\frac{\left(m_{a}^{4}+m_{b}^{4}\right)}{4\left(m_{a}^{2}-m_{b}^{2}\right)} \ln \left(\frac{m_{b}^{2}}{m_{a}^{2}}\right) \\
\tilde{\Pi}_{V-A}(0)= & m_{a} m_{b}\left(\operatorname{Div}+\ln \left(\frac{\mu^{2}}{m_{a} m_{b}}\right)+1+\frac{\left(m_{a}^{2}+m_{b}^{2}\right)}{2\left(m_{a}^{2}-m_{b}^{2}\right)} \ln \left(\frac{m_{b}^{2}}{m_{a}^{2}}\right)\right)
\end{aligned}
$$

The first and second derivatives are

$$
\begin{aligned}
\tilde{\Pi}_{V+A}^{\prime}(0)= & \left(\frac{1}{3} \operatorname{Div}+\ln \left(\frac{\mu^{2}}{m_{a} m_{b}}\right)\right)+\frac{m_{a}^{4}-8 m_{a}^{2} m_{b}^{2}+m_{b}^{4}}{9\left(m_{a}^{2}-m_{b}^{2}\right)^{2}}+ \\
& +\frac{\left(m_{a}^{2}+m_{b}^{2}\right)\left(m_{a}^{4}-4 m_{a}^{2} m_{b}^{2}+m_{b}^{4}\right)}{6\left(m_{a}^{2}-m_{b}^{2}\right)^{3}} \ln \left(\frac{m_{b}^{2}}{m_{a}^{2}}\right) \\
\tilde{\Pi}_{V-A}^{\prime}(0)= & m_{a} m_{b}\left(\frac{\left(m_{a}^{2}+m_{b}^{2}\right)}{2\left(m_{a}^{2}-m_{b}^{2}\right)}+\frac{m_{a}^{2} m_{b}^{2}}{\left(m_{a}^{2}-m_{b}^{2}\right)^{3}} \ln \left(\frac{m_{b}^{2}}{m_{a}^{2}}\right)\right),
\end{aligned}
$$

and

$$
\begin{aligned}
& \tilde{\Pi}_{V+A}^{\prime \prime}(0)=\frac{\left(m_{a}^{2}+m_{b}^{2}\right)\left(m_{a}^{4}-8 m_{a}^{2} m_{b}^{2}+m_{b}^{4}\right)}{4\left(m_{a}^{2}-m_{b}^{2}\right)^{4}}-\frac{3 m_{a}^{4} m_{b}^{4}}{\left(m_{a}^{2}-m_{b}^{2}\right)^{5}} \ln \left(\frac{m_{b}^{2}}{m_{a}^{2}}\right), \\
& \tilde{\Pi}_{V-A}^{\prime \prime}(0)=m_{a} m_{b}\left(\frac{\left(m_{a}^{4}+10 m_{a}^{2} m_{b}^{2}+m_{b}^{4}\right)}{3\left(m_{a}^{2}-m_{b}^{2}\right)^{4}}+\frac{2\left(m_{a}^{2}+m_{b}^{2}\right) m_{a}^{2} m_{b}^{2}}{2\left(m_{a}^{2}-m_{b}^{2}\right)^{5}} \ln \left(\frac{m_{b}^{2}}{m_{a}^{2}}\right)\right) .
\end{aligned}
$$


The values of the vacuum polarizations for identical masses $\left(m_{b}=m_{a}\right)$ are the smooth limits of the previous formulae and agree with direct calculation.

$$
\begin{array}{lll}
\tilde{\Pi}_{V+A}(0)=-m_{a}^{2} \operatorname{Div}-m_{a}^{2} \ln \left(\frac{\mu^{2}}{m_{a}^{2}}\right), & & \tilde{\Pi}_{V-A}(0)=m_{a}^{2} \operatorname{Div}+m_{a}^{2} \ln \left(\frac{\mu^{2}}{m_{a}^{2}}\right), \\
\tilde{\Pi}_{V+A}^{\prime}(0)=\frac{1}{3} \operatorname{Div}+\frac{1}{3} m_{a}^{2} \ln \left(\frac{\mu^{2}}{m_{a}^{2}}\right)-\frac{1}{6}, & & \tilde{\Pi}_{V-A}^{\prime}(0)=\frac{1}{6} \\
\tilde{\Pi}_{V+A}^{\prime \prime}(0)=\frac{1}{10 m_{a}^{2}}, & & \tilde{\Pi}_{V-A}^{\prime \prime}(0)=\frac{1}{30 m_{a}^{2}} .
\end{array}
$$

The new vector-like fermions contribute to the complete vacuum polarization as the sum of (15) and (16). We define

$$
\tilde{\Pi}_{V}\left(m_{a}, m_{b}, q^{2}\right)=\tilde{\Pi}_{V+A}\left(m_{a}, m_{b}, q^{2}\right)+\tilde{\Pi}_{V-A}\left(m_{a}, m_{b}, q^{2}\right) .
$$

In what follows the index $V$ is omitted we use $\tilde{\Pi}=\tilde{\Pi}_{V}$.

The $\hat{S}$ parameter (10) is then given by

$$
\hat{S}=\frac{g^{2}}{16 \pi^{2}}\left(+\tilde{\Pi}^{\prime}\left(M_{+}, M_{+}, 0\right)-c^{4} \tilde{\Pi}^{\prime}\left(M_{1}, M_{1}, 0\right)-s^{4} \tilde{\Pi}^{\prime}\left(M_{2}, M_{2}, 0\right)-2 s^{2} c^{2} \tilde{\Pi}^{\prime}\left(M_{2}, M_{1}, 0\right)\right) .
$$

The first three terms cancel the divergent contribution of the last one.

The $\hat{T}$ parameter (11) related to $\Delta \rho$ is also finite.

$$
\begin{aligned}
\hat{T}= & \frac{g^{2}}{M_{W}^{2} 16 \pi^{2}}\left[+\tilde{\Pi}\left(M_{+}, M_{+}, 0\right)+c^{4} \tilde{\Pi}\left(M_{1}, M_{1}, 0\right)+s^{4} \tilde{\Pi}\left(M_{2}, M_{2}, 0\right)+\right. \\
& \left.+2 s^{2} c^{2} \tilde{\Pi}\left(M_{2}, M_{1}, 0\right)-2 c^{2} \tilde{\Pi}\left(M_{+}, M_{1}, 0\right)-2 s^{2} \tilde{\Pi}\left(M_{+}, M_{2}, 0\right)\right] .
\end{aligned}
$$

The $Y$ and the $W$ parameters differ only in the coupling constants

$$
\begin{aligned}
Y=M_{W}^{2} \frac{g^{\prime 2}}{32 \pi^{2}} & \cdot\left[\tilde{\Pi}^{\prime \prime}\left(M_{+}, M_{+}, 0\right)+c^{4} \tilde{\Pi}^{\prime \prime}\left(M_{1}, M_{1}, 0\right)+\right. \\
+ & \left.s^{4} \tilde{\Pi}^{\prime \prime}\left(M_{2}, M_{2}, 0\right)+2 s^{2} c^{2} \tilde{\Pi}^{\prime \prime}\left(M_{2}, M_{1}, 0\right)\right], \\
W=M_{W}^{2} \frac{g^{2}}{32 \pi^{2}}[ & {\left[\tilde{\Pi}^{\prime \prime}\left(M_{+}, M_{+}, 0\right)+c^{4} \tilde{\Pi}^{\prime \prime}\left(M_{1}, M_{1}, 0\right)+\right.} \\
+ & \left.s^{4} \tilde{\Pi}^{\prime \prime}\left(M_{2}, M_{2}, 0\right)+2 s^{2} c^{2} \tilde{\Pi}^{\prime \prime}\left(M_{2}, M_{1}, 0\right)\right] .
\end{aligned}
$$

The first three terms in the parentheses give $W=\frac{g^{2}}{240 \pi^{2}} M_{W}^{2} \cdot\left(1 / M_{+}^{2}+c^{4} / M_{1}^{2}+s^{4} / M_{2}^{2}\right)$ in agreement with [17] taking into account that they considered Majorana fermions. The last term is the same order of magnitude in $M_{W} / M_{\{1,2,+\}}$. Here $W$ and $Y$ are always non-negative fulfilling the positivity constraints proven in [18].

\section{Numerical results}

There are 3 free parameter in the model to confront with the experiments: the two neutral masses $\left(M_{1,2}\right)$ and the mixing angle $\phi, s^{2}=\sin ^{2} \phi, c^{2}=\cos ^{2} \phi$. The mass of the 


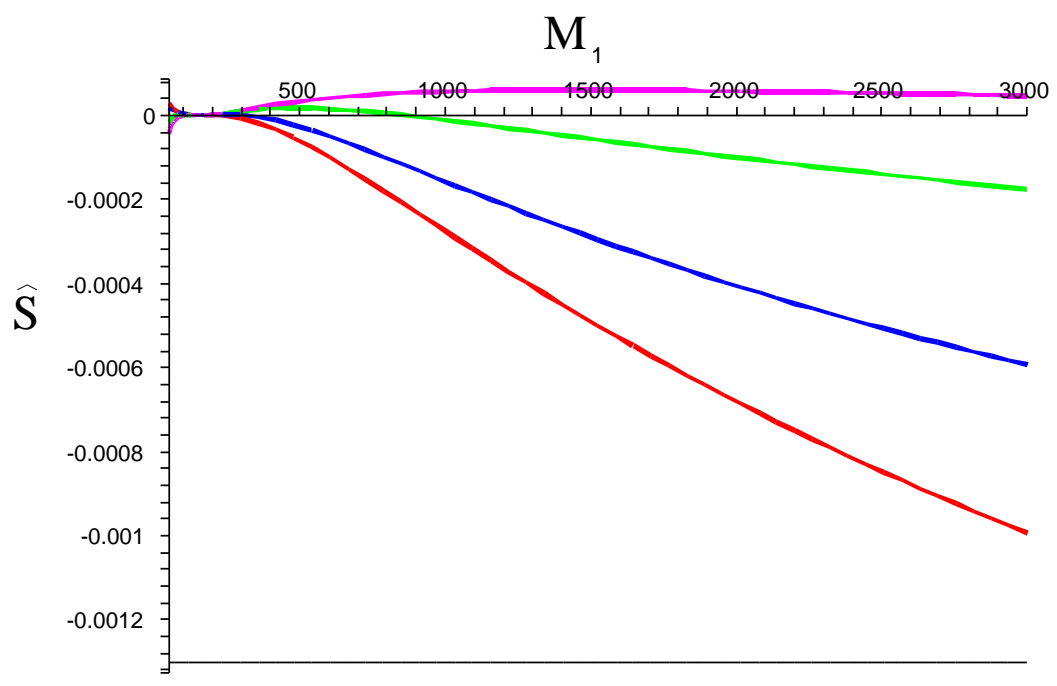

Figure 1: The $\hat{S}$ parameter vs. $M_{1}$ for $M_{2}=150 \mathrm{GeV}$ for $c^{2}=0.2,0.4,0.6,0.8$ respectively from bottom upwards (from red to magenta), the horizontal line is the $1 \sigma$ experimental lower bound.

charged fermion is given by $M_{+}=c^{2} M_{1}+s^{2} M_{2}$ (7). The new particles are expected to be heavier than approximately $100 \mathrm{GeV}$ from LEP1 and LEP2 as they have ordinary couplings with the gauge bosons. For relatively light new particles (with masses 100$150 \mathrm{GeV}$ ) the oblique parameters give rough estimate of the radiative corrections [17]. Replacing $M_{1} \leftrightarrow M_{2}$ and $c^{2} \leftrightarrow s^{2}=1-c^{2}$ gives the same oblique parameters. If there is no real mixing $c^{2}=0$ or 1 or if $M_{1}=M_{2}=M_{+}$then there is one degenerate vector-like fermion doublet and a decoupled singlet, $\hat{S}$ and $\hat{T}$ vanish explicitely. In this case the new sector does not violate $S U_{L}(2)$ and there is an exact custodial symmetry. Increasing the mass difference in the remnants of the original doublet by increasing the $\left|M_{1}-M_{2}\right|$ mass difference and/or moving away from the non-mixing case $c^{2}=0$, or 1 results in increasing $\hat{S}$ and $\hat{T}$. For small violation of the symmetries $\hat{S}$ and $\hat{T}$ are expected to be small.

The $\hat{S}, W, Y$ parameters are small for masses in the range from $100 \mathrm{GeV}$ up to few $\mathrm{TeV}$, the only exception is $\hat{T}(T)$, which is sensitive to the mass differences. These features were predicted using simple assumptions in [16]. We discuss in details the case of a light Higgs boson (Table 1).

Generally the $\hat{S}(S)$ parameter depends only on the masses of the new particles and the mixing angle, it contains no further dimensional parameter. For reasonable masses (below few $\mathrm{TeV}$ ) it is always in agreement with the $1 \sigma$ experimental bounds for $M_{1,2} \geq 100 \mathrm{GeV}$. See Fig. 1. For higher masses $|\hat{S}|(|S|)$ is even smaller. $S$ can have both signs. The $\hat{T}$ parameter (27) is more sensitive to the value of $M_{1,2}$. The mass 


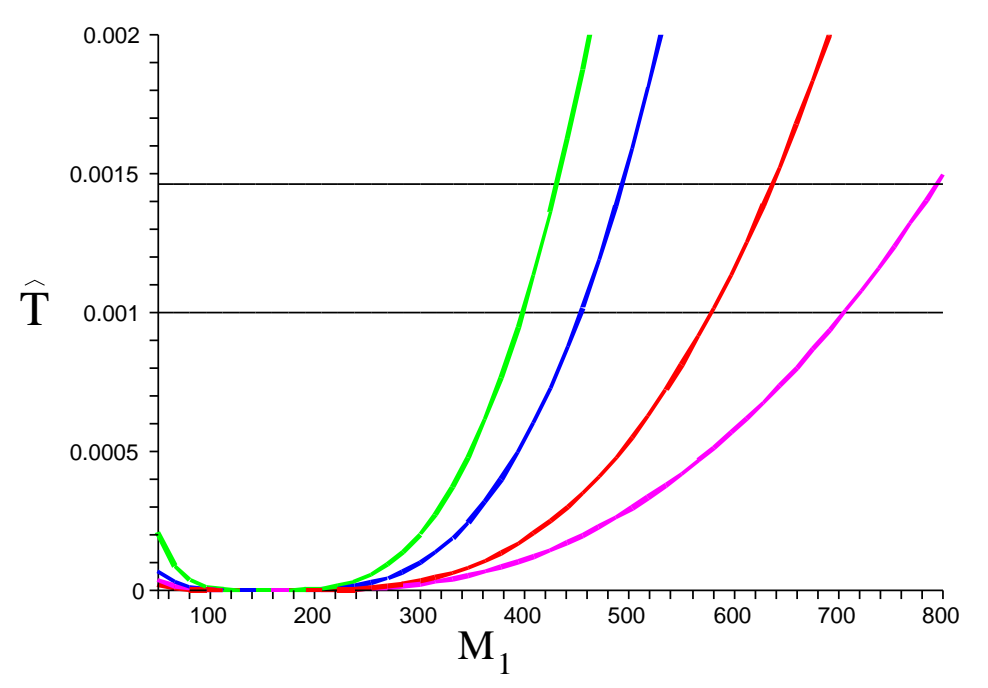

Figure 2: The $\hat{T}$ parameter vs. $M_{1}$ for $M_{2}=150, \mathrm{GeV}$ for $c^{2}=0.9,0.1,0.2,0.55$ from bottom upwards, the horizontal lines are the $1 \sigma$ and $1.6 \sigma$ experimental upper bounds.

difference of the new fermions must not exceed a critical value, $\left|M_{1}-M_{2}\right| \leq 250$ (400) for the mass of the lighter fermion 150 (500) GeV. The constraints are the strongest for $c^{2} \simeq 0.56$, below and above this mixing the absolute value of $\hat{T}$ decreases. For small mixing ( $c^{2}$ close to 0 or 1$)$ there are very weak or simply no constraints. Fig. 2 . shows as an example $\hat{T}$ for $M_{2}=150 \mathrm{GeV}$ as a function of $M_{1}$ for various mixings. $c^{2}=0$ (1) gives a horizontal line, $\hat{T}=0 . \hat{T}(T)$ is always positive allowing a heavy Higgs particle.

The $W$ parameter is sensitive to the ratio $M_{W}^{2} / M_{i}^{2}, i=1,2,+$. It is largest for relatively small masses approximately $(150 \mathrm{GeV})$, but $W$ is still well within the $1 \sigma$ experimental limits. For higher masses $W$ is even smaller. See Fig.3. The $Y$ parameter is the same function of the masses and mixing angles as $W$. The smaller gauge coupling multiplier provides weaker constraints.

If the Higgs is heavy, $M_{H}=800 \mathrm{GeV}$ (see Table 1.) the central value of $\hat{S}$ decreases compared to the light Higgs case. $\hat{S}$ and $W$ gives no constraints. At the same time the negative contribution of the light Higgs can be compensated by the new fermions with considerable mass difference for example $(150,400) \mathrm{GeV}$ or $(500,900) \mathrm{GeV}$. Nondegenerate vector-like fermions allow a space for heavy Higgs in the precision tests of the Standard Model.

In the fermion condensate model [8] the Higgs boson in (3) is a composite state of the new fermions. Gap equations were derived and solved for the parameters of the model. Applying further perturbative unitarity arguments constrains the model seriously, the charged particle mass must be relatively close to one of the neutral ones, e.g. $c^{2}$ must be close to 0 or 1 [16]. The solutions of the gap equations fulfill easily the experimental constraints on $\hat{S}$ and $\hat{T}$ due to the small mixing and the $W$ parameter is also safe. The solutions in [16] result that the oblique corrections do not constrain the 


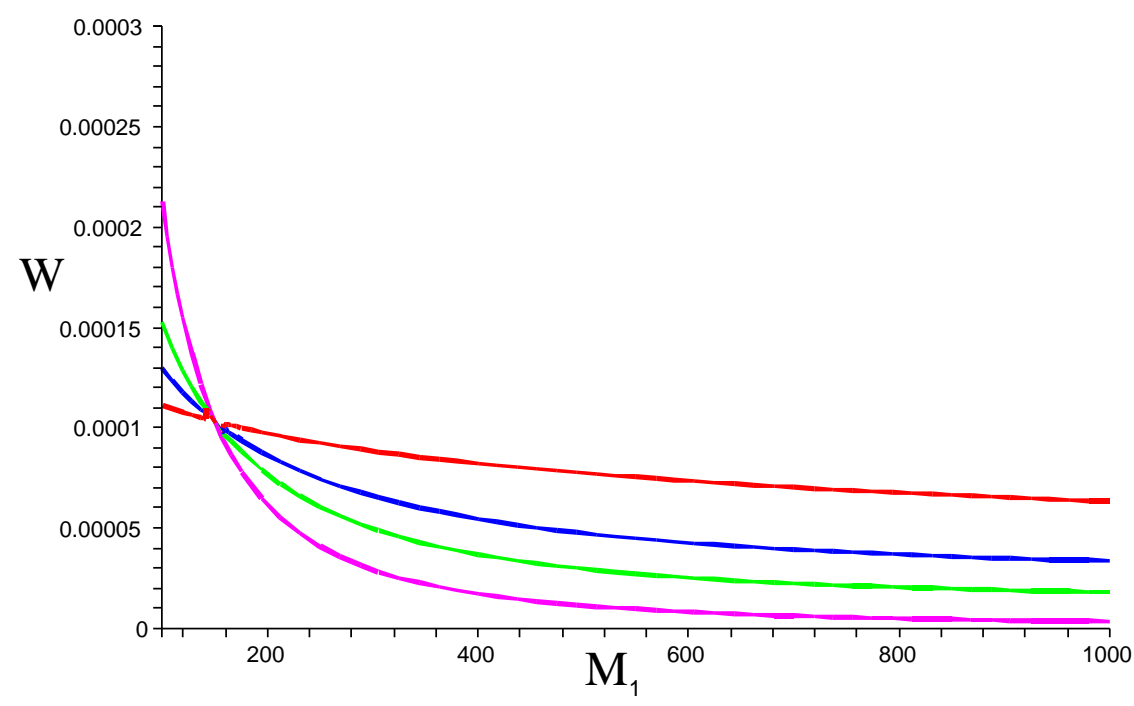

Figure 3: The $\mathrm{W}$ parameter vs. $M_{1}$ for $M_{2}=150 \mathrm{GeV}$ for $c^{2}=0.1,0.3,0.5,0.9$ respectively from top downwards at high $M_{1}$, the $1 \sigma$ experimental bound is at 0.0013 is outside the figure.

fermion condensate model even if the neutral masses $\left(M_{1,2}\right)$ are non-degenerate. The calculation presented in this paper shows that the fermion condensate model is less constrained than assumed by the naive estimates in [16]. The formulae derived here can be applied not just to [8], but to various models generating the Lagrangian (3)).

\section{Conclusions}

We have calculated the oblique corrections in an extension of the Standard Model based on vector-like weak singlet and doublet fermions. Due to non-diagonal mass terms (4) symmetry breaking mixing occurs between the singlet and the neutral component of the doublet. The oblique corrections were presented in the formalism of Barbieri et al. 12. There are four relevant parameters $\hat{S}, \hat{T}, W, Y$, and they are indeed in the same order of magnitude in the allowed mass range, as expected. $Y$ is the same function of the masses and mixing angle as $W$ with smaller coupling constant, but with weaker constraints therefore we kept $\hat{S}, \hat{T}, W$. The corrections depend on the new fermion masses $\left(M_{1,2}\right)$ and the mixing angle. The $\hat{S}, W$ parameters are always in agreement with experiment for masses below few $\mathrm{TeV}$. The $\hat{T}(T)$ parameter measures the custodial symmetry breaking, the custodial symmetry is exact in the new sector if there is no physical mixing: $c^{2}=0,1$ or $M_{1}=M_{2}$. Depending on the mixing angle it allows in the most stringent case for $c^{2} \simeq 0.56$ a maximal mass difference $\left|M_{1}-M_{2}\right| \lesssim 250 \mathrm{GeV}$ at $1 \sigma$ for relatively small ligter neutral mass $(\sim 150 \mathrm{GeV})$, higher mass difference is allowed for higher $M_{1,2}$ masses or differen mixing. This 
extension/modification nicely accommodates a heavy Higgs in the Standard Model. The lightest new fermion is stable and a good dark matter candidate. The model can be tested at LHC in the Drell-Yan process [8] or via jetmass analysis [9]. Nearing the completion of our work we received a preprint which deals with similar topic, but with different fermion representation, approach and mixing allowed with the standard fermions [19].

\section{Acknowledgment}

The authors thank George Pócsik for valuable discussion.

\section{References}

[1] T. Appelquist, H-C. Cheng, B. A. Dobrescu, Bounds on universal extra dimensions, Phys. Rev. D 64 (2001) 035002 arXiv:hep-ph/0012100|.

[2] N. Arkani-Hamed, A. G. Cohen, E. Katz and A. E. Nelson, The littlest Higgs J. High Energy Phys. 0207 (2002) 034 |arXiv:hep-ph/0206021|; N. Arkani-Hamed, A. G. Cohen, E. Katz, A. E. Nelson, T. Gregoire, Jay G. Wacker, 'The minimal moose for a little Higgs, J. High Energy Phys. 0208 (2002) 021 arXiv:hep-ph/0206020|.

[3] Riccardo Barbieri, Lawrence J. Hall, Vyacheslav S. Rychkov, Improved naturalness with a heavy Higgs: An alternative road to LHC physics, Phys. Rev. D D74 (2006) 015007 arXiv:hep-ph/0603188

[4] R. Enberg, P. J. Fox, L. J. Hall, A. Y. Papaioannou and M. Papucci, LHC and Dark Matter Signals of Improved Naturalness, J. High Energy Phys. 0711 (2007) 014 (2007) arXiv:0706.0918 [hep-ph]]; Rakhi Mahbubani, Leonardo Senatore, 'The minimal model for dark matter and unification, Phys. Rev. D 73 (2006) :043510 arXiv:hep-ph/0510064|; M. Cirelli, N. Fornengo and A. Strumia, Minimal dark matter, Nucl. Phys. B 753 (2006) 178 arXiv:hep-ph/0512090.

[5] F. D'Eramo, Dark matter and Higgs boson physics, Phys. Rev. D 76 (2007) :083522 [arXiv:0705.4493 [hep-ph]].

[6] W.A. Bardeen, C.T. Hill and M. Lindner, Minimal Dynamical Symmetry Breaking of the Standard Model Phys. Rev. D 41 (1990) 1647 ; C.T. Hill, opcolor: Top quark condensation in a gauge extension of the standard model, Phys. Lett. B 266 (1991) 419; M. Lindner and D. Ross, Top condensation from very massive strongly coupled gauge bosons, Nucl. Phys. B 370 (1992) 30;

Bogdan A. Dobrescu and Christopher T. Hill, Electroweak symmetry breaking via top condensation seesaw, Phys. Rev. Lett. 81 (1998) 2634

arXiv:hep-ph/9712319. 
[7] E. Ma, Increasing $R_{b}$ and Decreasing $R_{c}$ with New Heavy Quarks, Phys. Rev. D 53 (1996) 2276 |arXiv:hep-ph/9510289|; P. Bamert, C.P. Burgess, J.M. Cline, D. London, E. Nardi, $R_{b}$ and New Physics: A Comprehensive Analysis, Phys. Rev. D 54 (1996) 4275 arXiv:hep-ph/9602438..

[8] G. Cynolter, E. Lendvai and G. Pócsik, Fermion condensate model of electroweak interactions, Eur. Phys. J. C 46 (2006) 545 arXiv:hep-ph/0509230.

[9] Witold Skiba, David Tucker-Smith, Using Jet Mass To Discover Vector Quarks At The Lhc Phys. Rev. D 75 (2007) :115010 arXiv:hep-ph/0701247.

[10] M. E. Peskin and T.Takeuchi, Estimation Of Oblique Electroweak Corrections, Phys. Rev. D 46, (1992) 381.

[11] W-M Yao et al, Review of particle physics, J. Phys. G 33 (2006) 1.

[12] R. Barbieri, A. Pomarol, R. Rattazzi and A. Strumia, Electroweak symmetry breaking after LEP1 and LEP2, Nucl. Phys. B 703 (2004) 127 arXiv:hep-ph/0405040.

[13] I. Maksymyk, C.P. Burgess and David London, Beyond S, T and U, Phys. Rev. D 50 (1994) 529 |arXiv:hep-ph/9306267| ; G. Altarelli, R. Barbieri and S. Jadach, Toward A Model Independent Analysis Of Electroweak Data Nucl. Phys. B 369 (1992) 3.

[14] L. Lavoura and J. P. Silva, The Oblique Corrections From Vector - Like Singlet And Doublet Quarks, Phys. Rev. D 47 (1993) 2046.

[15] M. Chen and S. Dawson, One-loop radiative corrections to the rho parameter in the littlest Higgs model, Phys. Rev. D 70 (2004) 015003 arXiv:hep-ph/0311032.

[16] G. Cynolter, E. Lendvai, Gap Equations and Electroweak Symmetry Breaking, J. Phys. G 34 (2007) 1711 arXiv:hep-ph/0607021.

[17] G. Marandella, C. Schappacher and A. Strumia, Supersymmetry and precision data after LEP2, Nucl. Phys. B 715 (2005) 173 |arXiv:hep-ph/0502095|.

[18] G. Cacciapaglia, C. Csaki, G. Marandella and A. Strumia, The minimal set of electroweak precision parameters, Phys. Rev. D 74 (2006) 033011 arXiv:hep-ph/0604111.

[19] F. del Aguila, J. de Blas and M. Perez-Victoria, Effects Of New Leptons In Electroweak Precision Data, arXiv:0803.4008. 